\title{
A 50 AÑOS DEL ESTABLECIMIENTO DE LA EMPRESA VOLKSWAGEN DE MÉXICO. Su impacto en el paisaje cultural del valle de Puebla-Tlaxcala
}

\author{
Mariano TORRES BAUTISTA \\ Benemérita Universidad Autónoma de Puebla (México) \\ mariano.torres@gmail.com
}

\begin{abstract}
A 50 YEARS OF ESTABLISHMENT OF THE COMPANY VOLKSWAGEN-MEXICO. It's impact on the cultural landscape of the valley of Puebla-Tlaxcala
\end{abstract}

Resumen: Este ensayo explora los cambios producidos por el establecimiento de una corporación multinacional en medio de comunidades agrícolas estancadas tecnológicamente. Presenta algunos de los resultados inesperados por políticas industrializadoras desarrollistas que nunca atienden las necesidades de desarrollo de las comunidades agrícolas y pretenden como única alternativa de desarrollo la transformación del campesinado en obrero industrial.

Abstract: This paper explores the changes that occurred in Puebla, Mexico, after the establishment of Volkswagen, a german multinational corporation, in the technologically stagnant agricultural communities of this mexican State. Industrialization policies, which did not focus on the needs of agricultural communities, produced unexpected results such as the transformation of the peasantry into an industrial working class.

Palabras clave: Políticas públicas. Campesinado. Industrialización. Paisaje cultural. México central Public policies. Peasantry. Industrialization. Cultural landscape. Central Mexico 


\section{Introducción}

Evidentemente, son muchos los aspectos de gran relevancia que parten del establecimiento y desarrollo de una gran e importante industria como es la automotriz Volkswagen dentro de la propia trayectoria de las comunidades agrícolas que en el Estado de Puebla, en la región centro oriente de México albergaron su construcción. El impacto en las formas de organización social, de los esquemas culturales (en particular de la cultura del trabajo) sobre todo, partieron de un embrión concebido con unos fines muy específicos que, nos obstante, llegaron a lo que hoy es una gran empresa tecnológicamente vanguardista a escala mundial y se ha posicionado como la armadora más grande del consorcio VW en todo el continente americano. Trataremos de reunir aquellos de mayor impacto dentro del contexto social en el que se inscribe.

La historia del automóvil como producto industrial y forma de potencializar la actividad humana es muy extensa. Sin embargo, el auto con la mayor amplitud de difusión, tanto en los mercados como en la historiografía, es inequívocamente el modelo "escarabajo", diseñado por Ferdinand Porsche en 1938. Este ingenio tecnológico se concibió buscando satisfacer una de las premisas del ejercicio del poder del socialismo nacionalista alemán: dotar a las bases sociales medias de un "Volksauto" un auto para el pueblo. Desde el multicitado artículo: "Volkswagen Abroad", hasta la reciente obra de Bernhard Rieger: The People's $\mathrm{Car}^{2}$ pasando por la impactante obra: Place of Remembrance of the History of Forced Labor for the Volkswagen Factory, ${ }^{3}$ son docenas de libros escritos a propósito del "Volksauto", sin contar las más de 100 apariciones en revistas automovilísticas. Esta numerosa bibliografía y hemerografía tiene significación por sí misma. Más que una efímera imagen de poder y sin las exclusivas líneas de los autos de gran lujo, el "auto para el pueblo" cumplió con otras premisas de significación técnica: relación precio-calidad, bajos costos de mantenimiento y alto rendimiento. Una vez cubiertas esas expectativas, los años siguientes fue cumpliendo gradualmente con mejoras en su diseño y los requerimientos de seguridad hasta que sus evolucionadas formas ya no lograron pasar este último estándar suficientemente. Luego de que este modelo fuese perdiendo terreno ante otros autos de clase económica y fuese producido exclusivamente en la planta armadora de Puebla, la estocada final la daría el entonces Jefe de gobierno de la ciudad de México: Cuauhtémoc Cárdenas, al prohibir el uso de autos de dos puertas como taxis, en ese que era el principal mercado del país. No obstante, el emblemático auto que daba "fuerza desde la alegría" a las nuevas capas sociales, había superado las 16,255,500 unidades producidas en Alemania hasta 1978 y en total 21,529,464 al salir de la planta de "Volkswagen de México, Sociedad Anónima de Capital Variable, el último "Vochito" el 30 de julio de 2003 con música de mariachis al toque de "las golondrinas", obra clásica en despedidas festivas.

No es el objetivo de este ensayo hacer un resumen de la historia de este ingenio tecnológico. No obstante, cabe destacar como factores claves de la expansión de la compañía -que tuvo su origen precisamente en el modelo escarabajo- entre otros: la planeación estratégica para la construcción masiva de un producto nuevo, desarrollar un modelo de automóvil para una sociedad cambiante; un vehículo familiar para 4 personas distinto al concepto elitista del auto como símbolo de prestigio social que prevalecía hasta entonces entre los fabricantes de automóviles europeos. De esta manera se escogió por razones técnicas al pequeño pueblo de Fallersleben, en el condado de Schloss Wolfsburg, a orillas del Canal Mitelland. Es así como la nueva planta contó con una vía fluvial navegable que posibilitó el suministro

1 "Volkswagen Abroad", en Autocar, August 10, 1951.

2 RIEGER, Bernhard. The people's car: a global history of the Volkswagen Beetle. Harvard, Massachusets, 2013 3 Volkswagenwerk, Place of Remembrance of the History of Forced Labor for the Volkswagen Factory. Wolfburg, 2000. 
económico y masivo de insumos, dispuso igualmente de una central hidroeléctrica propia para proporcionar energía también con costos razonables, tanto para la fábrica como a la ciudad que ya se preveía iba a surgir por la necesaria colonización de sus trabajadores. ${ }^{4} \mathrm{La}$ otra razón, de tipo político, que definió esta localización fue el que esta gran industria estatal coadyuvase a solucionar los problemas de pobreza y desempleo de la Baja Sajonia, ya que lugares con las características geográficas mencionadas se encontraban en ese momento en muchas partes de Alemania.

Vemos así que el impacto de una empresa de este tipo no es algo fortuito, de gran envergadura, y debe estar en las previsiones del surgimiento de este tipo de compañías. Otra de las premisas que acompañarán este ejercicio del poder -tanto político como tecnológico- fue el pensar desde el principio en un desarrollo a partir de la fabricación de un solo modelo de automóvil, con solo algunos cambios en su diseño que mejoraran gradualmente la calidad. Un producto con todas estas características estaría en la capacidad de cumplir las expectativas de una sociedad en metamorfosis, gracias al amplio desarrollo industrial alemán que abrió paso a una clase social distinta de aquella retratada en la historia de la revolución industrial británica. ${ }^{5}$ La nueva fábrica de Wolfburg fue inaugurada oficialmente el 26 de mayo de 1938. A pesar de la cuidadosa planeación con la que se había concebido, el inicio todavía no explicado de la $2^{a}$ Guerra Mundial el $1^{\circ}$ de septiembre de 1939 , acabó con las expectativas de este y otros proyectos del vertiginoso desarrollo de Alemania. Con la caída del poder del socialismo nacionalista alemán, en marzo de 1946 la fábrica de Wolfburg, -bajo el dominio británico y la administración del Mayor Ivan Hirst- logra la producción del "escarabajo" número $1,000 .{ }^{6}$ No obstante, fue al volver a propiedad alemana el $1^{\circ}$ de enero de 1948, con Heinrich Nordhoff como director, que se da la proyección internacional del "escarabajo" convirtiéndose en un emblema más de la tecnicidad alemana de los nuevos tiempos. Nordhoff calculó visionariamente que la exportación era la única manera de aumentar de manera sostenida la producción salida de las enormes naves construidas ex profeso. Su siguiente premisa fue que, para poder tener éxito en el exterior, era necesario ofrecer un modelo exportable con un grado de calidad más alto que el modelo básico. Fue en la década de los 50 cuando se consolidaría a nivel mundial el "escarabajo". En 1950 se logró producir lo que hoy nos parecería la modesta cantidad de 312 vehículos del modelo "Export" cuya llegada a los EE.UU. logró salir al paso de otro gran obstáculo: la mala imagen que de Alemania se había difundido en ese país para justificar la entrada estadounidense en la guerra que Gran Bretaña sostenía en el continente europeo, y el consabido sacrificio de millones de jóvenes estadounidenses, a partir de una estrategia que el mismo Churchill había considerado como brutal y nunca aprobó del todo. No obstante, la aceptación en el que ya era el principal mercado mundial del automóvil, permitió que en tan solo 5 años se saltara a una producción de 1,000 Volkswagen diarios lográndose además, el 5 de agosto de 1955, la producción del primer millón del emblemático modelo.

A principios de la década de los sesenta del siglo pasado, el problema principal del establecimiento de un complejo industrial con un grado de evolución tecnológica altísimo

4 VW Chronik. Historische Notate. Schiftenreihe der historischen kommunikation der Volkswagen A. G. Wolfsburg, 2003.

5 El prototipo del modelo escarabajo Serie 38 pasó a llamarse Kdf-Wagen que significaba "a la fuerza por la alegría", solo estaba disponible con un color y solamente se podía adquirir financiando previamente su producción, se había de pagar antes de su entrega reuniendo unos cupones especiales por valor de 5 marcos, al llegar a los 90 ya se podía disponer del coche pagando 50 marcos más en el momento de su entrega y otros 200 por dos años de seguro. El contrato no se podía rescindir y en caso de no pagar uno de los plazos, se perdía todo lo acumulado, pese a todas estas duras condiciones el pedido inicial fue realizado por ¡336.600 personas! Cf. http:// www.avwc.org/castella/historiavw.htm consultado el 28 de julio de 2014.

6 RICHTER, Ralf. Ivan Hirst, britischer offizier und manager des volkswagenaufbaus. Historische Notate Schiftenreihe des Unternehmenschaft der Volkswagen AG, Wolfburg, 2003. 
y constante, estuvo en la gran distancia existente entre este patrón industrial y la forma de vida de las comunidades agrícolas tecnológicamente estancadas del México central que lo iban a rodear. Además de la apertura en un mercado nuevo y el desarrollo de la novísima infraestructura necesaria, hubo que hacer frente a las necesidades de trabajo altamente especializado de una armadora automotriz que buscó abrirse paso en un país dominado por la industria automotriz americana del momento. Además, en México prevalecía el concepto de automóvil como bien de consumo de alto valor, empatado todavía con un imaginario social que lo veía mucho más como un objeto de prestigio que como un medio de solucionar las necesidades de movilidad de la clase media, capa social emergente y poco visible hasta esos momentos en un país subdesarrollado.

Evidentemente, el reto que implicó la implantación de esta gran industria en un contexto altamente tradicional, es una fuente inexplorada de análisis de procesos de industrialización acelerado, un campo de estudio social e histórico de gran relevancia. Nuestro trabajo es un esfuerzo por asociar la investigación social implicada, privilegiando la historia del contexto espacial insuficientemente explotada, apoyándola con investigación etnográfica y antropológica. Además, es considerable la importancia del trabajo de campo y la historia oral que permiten comprender las profundas mutaciones experimentadas en el paisaje cultural contiguo a esta gran industria. Para abordar esta problemática existe un sinfín de interrogantes. Por ejemplo: ¿Cuáles son las posibilidades para la potencialización del conocimiento de este fenómeno con un enfoque y análisis interdisciplinario? ¿En qué medida la etnografía, la historia oral, la antropología del espacio, la sociología del trabajo, la geografía cultural; por mencionar las principales disciplinas, permiten recuperar los vestigios de la actividad de esta industria al momento de su establecimiento hace 50 años? Un complejo productivo de las dimensiones de la armadora automotriz VW hoy en día, es parte substancial del patrimonio industrial del valle poblano-tlaxcalteca, en la porción centro oriente de la república mexicana. No obstante, la compañía Volkswagen de México, difiere notablemente del antiguo patrimonio industrial basado en la industria textil, harinera y de bienes de consumo de los periodos precedentes ${ }^{7}$ : (Un área de trabajo enorme, cultura material de diferente índole, procesos mucho más complejos y cambiantes como una constante, involucramiento de sectores de actividad de una gama mayor). ¿Cómo podemos aprehender todos esos cambios?

\section{Lo novedoso y sus beneficios}

En 1998, A los 35 años de establecimiento de la empresa VW de México, S. A. de C.V. se publicó una obra excepcional basada en testimonios de los propios actores sociales involucrados: Una historia sin fin. Escrita por Gerhard Schreiber, Eduardo Calderón Sotomayor y Hermann Schnell. Este es un interesante trabajo no tan sólo como obra conmemorativa y como historia de empresa, sino que permite descubrir los orígenes de la armadora automotriz más grande del continente americano, donde las cosas no son lo que empíricamente parecería producto de una decisión corporativa o estrategias industriales. Dicha obra cuenta, entre otras características, con los testimonios de los actores sociales involucrados que todavía vivían al momento de ser elaborada. A grandes rasgos, se percibe que su establecimiento más que una decisión a muy alto nivel, estuvo basada en la voluntad de un artífice alemán interesado en dar a México, el país de acogida, una empresa alemana. Este también visionario actor social sin duda nunca se imaginó los alcances de su esfuerzo, ni como diferentes coyunturas favorecieron el desarrollo de esta empresa modesta en su punto de partida. En este caso se cumple lo que A. F. Garçon considera « ... la idea visionaria de los beneficios esperados de lo novedoso... ». ${ }^{8}$ ¿De que otra forma se puede catalogar la tenacidad del Prín-

7 Cf. TORRES BAUTISTA, Mariano. El origen de la industrialización de Puebla. Claves Latinoamericanas-El Colegio de Puebla, 1995, México.

8 GARÇON, Anne Françoise. L'Imaginaire et la pensée technique. Un approche historique, XVIe-XXe siècle. 
cipe Alfonso von Hohenlohe al conseguir la cesión de derechos de importación de los autos VW de manos de quien de alguna manera los había obtenido y afortunadamente nunca ejercido; un político de los años 50, el General Martínez. Este último, quien nunca había visto un "Escarabajo" y tenía la mentalidad propia de la capa social de generales emanados de la revolución mexicana, era poseedor de "...un enorme Cadillac, con accesorios, chapeado

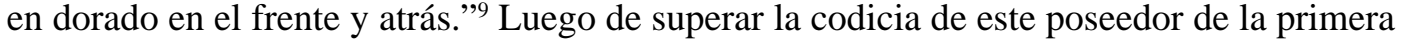
concesión el cual, al ver una fotografía del pequeño auto pensado para una capa social casi invisible en México a mediados del siglo XX, cedió los derechos no ejercidos una vez que sus pretensiones de beneficio sin haber realizado ninguna inversión entraron en razón. El Príncipe logró a su vez del importador general, el señor Ernesto Krause, un nuevo contrato. La dedicación prestada a su empresa costó igualmente al Príncipe Alfonso von Hohenlohe el abandono de su esposa, la también princesa Ira von Fürstemberg, más conocida por la frase que acuñó luego del tedioso trato con las damas de la alta sociedad mexicana de la época quienes, para ella; "Solo sabían hablar de hijos y criadas"10. El Príncipe Alfonso von Hohenlohe también logró captar la atención de la clientela de tan novedoso producto cuando consigue la adquisición de un "Escarabajo" por parte de la Presidenta del Jockey Club. Para la aceptación por parte del gran público fue decisiva la participación de 4 autos que hicieron completo el recorrido de la carrera Panamericana desde la frontera sur con Guatemala hasta la frontera norte con los Estados Unidos, demostrando la fortaleza de tan "pequeño" auto en el montañoso y cambiante territorio mexicano. Estos hechos, aparentemente discordantes, son un atisbo a las circunstancias que hicieron posible la introducción de los autos VW en México desde 1954, debido más los beneficios esperados por el Príncipe von Hohenlohe de lo novedoso, que a una decisión corporativa desde la sede de Wolfburg.

\section{Dominar el entorno inmediato así como a los hombres que lo habitan}

¿Porqué se escogió al pequeño pueblo de San Lorenzo Almecatla, -en el municipio de Cuautlancingo, a orillas de la casi recién inaugurada autopista México-Puebla que se prolongaba hasta el Puerto artificial de Veracruz, para la construcción de la que ahora es la armadora de autos más grande del continente americano? Evidentemente, fue de esta conexión carretera entre el principal puerto del país con su principal mercado como la nueva planta pudo contar con una conexión para el suministro masivo de insumos provenientes originalmente de Alemania. Esta fue verdaderamente una situación análoga a la de la casa matriz, ya que en esta zona no se encuentran vías navegables y, al igual que su sector agropecuario, los gobiernos "emanados de la Revolución" habían dejaron por otros intereses la modernización de la red ferroviaria preexistente. También es importante el hecho de que en el mismo sentido de este eje carretero, paralelamente corren líneas de transmisión eléctrica de alta tensión que permitieron también contar con energía a costos razonables; tanto para la fábrica, como para la nueva urbanización que evidentemente iba a surgir por la necesaria colonización de sus trabajadores. Tratándose de la implantación de VW, en un país donde lo que menos se practica es la previsión; no todas las premisas de esta gran armadora de autos compactos fueron contempladas desde el principio.

Siguiendo otra vez la relación entre imaginario y pensamiento técnico, se encuentra en este caso la utilización de diferentes eufemismos para hacer socialmente aceptable la dominación del entorno y sus habitantes. En primer lugar se encuentra aquí un conjunto de continuidades en el espíritu de «modernización » de las capas sociales en el poder. La importación de autos VW, su posterior armado y desde 1964 ensamblado con 60\% de pie-

Classiques Garnier, Histoire des techniques 3, Paris, 2012, p 32

9 SCHREIBER, Gerhard; CALDERÓN SOTOTMAYOR, Eduardo: SCHNELLE, Hermann. Una Historia sin fin. Eine Geschichte ohne ende. A Never-Ending Story. Puebla, México: Volkswagen de México, 1998, p. 30 10 LOAEZA, Guadalupe. Los secretos de las niñas bien. Ed. Océano, México, 2012 
zas fabricadas, vino a cumplir con una de las tareas enarboladas en el discurso oficial de los gobiernos "emanados de la revolución mexicana". Es un caso similar al de la creación de Ciudad Sahagún y el establecimiento de la empresa estatal "Diesel Nacional" (DINA) el 28 de julio de 1951 en la deprimida zona conocida como los "Llanos de Apan" en el Estado de Hidalgo, una de las entidades más pobres de México. En este caso se contrató asesoría técnica de la Fabbrica Automobilistica Italiana a Torino (FIAT). Al año siguiente se creó la Constructora Nacional de Carros de Ferrocarril, y en 1954 se construyó la Fábrica Nacional de Maquinaria Textil "Toyoda de México”, misma que pasó a denominarse Siderúrgica Nacional (Sidena). ${ }^{11}$ El plan era no solo construir un complejo industrial moderno, sino además incidir en el salto social, gracias a los beneficios de la técnica, para una de las regiones semiáridas más pobres del país, donde la incautación de las haciendas pulqueras, como en otras zonas del país, habían dado al traste con la dinámica de la economía agrícola comercial basada en la producción del "Pulque", la cerveza indígena de Mesoamérica; en este caso adaptada a un clima frío semidesértico, como es el del valle de Apan. Al igual que pasó en toda la región central de México, en vez de crear explotaciones rurales planeadas a partir de conocimientos técnicos realistas, empoderar la tecnicidad campesina, capacitar en las mejores técnicas agrícolas disponibles, adaptables a los tipos de suelo y clima, superar los retos de una sociedad que conocía las limitaciones de su economía agraria, ${ }^{12}$ se optó por el abandono del mundo rural para impulsar una industrialización desvinculada del resto del sistema económico nacional.

En el caso del establecimiento de Volkswagen de México tenemos un fenómeno curioso, casi providencial, de solución a la incapacidad de la clase política mexicana para cumplir con su discurso de reivindicación social y modernización "revolucionaria". Ya dijimos que aquí fue la voluntad del Príncipe de Alfonso Hohenlohe y de Ernesto Krause quienes logran, tanto la introducción del modelo "Escarabajo" en México, como superar las dudas de Heinrich Nordhoff y el consejo directivo de Volkswagen A. G. ante los conflictos y decepcionantes resultados de la planta instalada en Brasil en $1953^{13}$ que habían creado desconfianza en la calidad de la producción realizada fuera de Wolfburg. Es de esta manera como la tenacidad de estos alemanes residentes en México y la participación de la inversión externa crean una alternativa económica para Puebla, la ciudad industrial más antigua del país en franco declive una vez que su estancada producción textil se movía como elefante viejo, caminaba si pero solo rumbo al cementerio. ${ }^{14}$ También es de destacar que el movimiento social que caracterizaba a la efervescente sociedad poblana de la década de los 1960 no fue obstáculo para la implantación y desarrollo de esta nueva actividad industrial. ${ }^{15}$ Esto es característico de la cultura laboral alemana que, a diferencia del discurso del miedo del neoliberalismo actual, no ve en la organización sindical y en la manifestación del descontento social justificado un peligro, sino que desde décadas atrás prefiere el concurso con interlocutores responsables, honestos y fuertes.

11 MOIRÓN GÁLVEZ, Sara. Crónica de Ciudad Sahagún, 1972, 188 pags.

12 TORRES BAUTISTA, Mariano. "Colapso de un régimen y reorganización del estado revolucionario en México: cultura política heredada y fundamentos económicos distorsionados (1915-2006) " En prensa.

13 BERUMEN ARELLANO, Sergio Alejandro. (Coord.) Cambio tecnológico e innovación en las empresas. ESIC Editorial, Madrid, 2007, pag. 49

14 A manera de ejemplo tenemos que la participación de la industria textil mientras en 1970 era del $2.3 \%$ del PIB, en 1980 fue de sólo el 1.4\%. Elaborado a partir de la Memoria estadística de la Cámara Nacional de la Industria Textil. México por PORTOS, Irma. La industria textil en México y Brasil: dos vías nacionales de desarrollo. UNAM- Juan Pablos Editores, México, 2008.

15 CASTELLANOS, Laura. México armado 1943-1981. Editorial Era, México, 2007. 


\section{¿La lucha por "la tierra" frena inversiones?}

Otro de los ejercicios del poder se manifestará en una acción crucial para el establecimiento de esta gran industria en la región: la legitimación del régimen de propiedad de las 196 hectáreas compradas al pueblo de San Lorenzo Almecatla, más una franja adquirida del contiguo pueblo de San Francisco Ocotlán, para conformar las 201 hectáreas, 77 áreas y 55.16 centiáreas en las que se estableció la nueva planta de Volkswagen de México. San Lorenzo era una comunidad rural que, como muchas en esta zona y en el vecino Estado de Tlaxcala antes de la revolución de 1910-1917, habían tenido una expansión territorial a partir de sus propios recursos. Aunque existe en el Archivo General de Notarías de Puebla el protocolo de la Notaría $\mathrm{N}^{\circ}$ 5, fechado el 13 de agosto de 1874, que testimonia la escritura de compra del Rancho de San Juan Tepeyahualco por 187 vecinos del pueblo de San Lorenzo Almecatla al sr. Cayetano Amieva; el gobierno del Estado de Puebla se ocupó de legitimar esa propiedad "regularizando", para en el mismo acto decretar la expropiación de los bienes comunales de la población rural citada. Parecía como si la propiedad comunal conquistada antes de la época de los gobiernos "emanados de la revolución" no tuviera valor contractual; una fórmula que parecía expresarse en una fórmula del ejercicio del poder que establecía como consigna: "Si yo no te lo dí yo, no es tuyo". El eufemismo que acompañó este acto de poder se expresó, como en otros casos, a partir de la manipulación del confuso pero siempre útil lenguaje jurídico:

La Dirección General de Asuntos Jurídicos opinó que la documentación aportada por la comunidad promovente acredita los derechos de la misma, sobre la superficie de 662-85-95 Hs., de los terrenos comunales cuyo reconocimiento y titulación se pretende; de las cuales el núcleo solicitante ha venido poseyendo con carácter comunal en forma pacífica, pública continua y a título de dueño desde tiempos inmemoriales 466-85-95 Has. y el resto de la superficie o sean 196-00-00 Has., ocupadas por la fábrica de automóviles Volkswagen de México, S. A. de C. V., y para cuya regularización el Gobierno del Estado de Puebla, con fecha 25 de noviembre de 1974, solicitó su expropiación, solicitud que a su vez hizo suya con fecha 23 de enero de 1975, el Banco Nacional de Obras y Servicios Públicos. (...) al efecto y las opiniones del Instituto Nacional Indigenista, de la Delegación Agraria y de la Dirección General de Bienes Comunales son en el sentido de que es procedente el reconocimiento y titulación de los terrenos comunales del poblado mencionado, por haberse reunido los requisitos que señalan la Ley Federal de Reforma Agraria y el Reglamento respectivo. ${ }^{16}$

Esta generosa acción de gobierno terminó con la incertidumbre jurídica que durante 10 años se cernía sobre la propiedad territorial ocupada para la cuantiosa inversión realizada por Volkswagen. ${ }^{17} \mathrm{Al}$ momento del "Reconocimiento y titulación de bienes comunales", la

16 Secretaría de la Reforma Agraria (Hoy Secretaría de Territorios Agrarios) Expediente de Confirmación de Bienes Comunales a los vecinos de San Lorenzo Almecatla, Municipio de Cuautlancingo, Estado de Puebla. Resolución Presidencial del 22 de febrero de 1975. Diario Oficial de la Federación, 6 de marzo de 1975. Las cursivas de énfasis son nuestras.

17 Aunque no es muy escrupulosa en su información, según notamos en la lectura de la obra de Yolanda Montiel, esta autora señala en 1,000,000,000.00 de pesos la cifra de inversión del consorcio VW en Puebla, cifra que permite dimensionar a grandes rasgos el monto de lo que estaba en juego. Montiel, Yolanda Proceso de trabajo, acción sindical y nuevas tecnologías en Volkswagen de México. Centro de Investigaciones y Estudios superiores en Antropología Social, México, 1991, p. 43 
acción gubernamental consideró un censo para San Lorenzo Almecatla que agrupaba 570 comuneros de los cuales 123 eran mujeres. ${ }^{18}$

\section{Un campo de investigación inexplorado}

Para estudiar el impacto en el paisaje regional, en los patrones de vida comunitarios, familiares, las actitudes ante la modernización técnica y las relaciones sociales en el caso de estudio; se ha tratado de acceder a una fuente poco accesible, de limitada magnitud, pero de una riqueza inigualable: los archivos de los municipios contiguos a la planta, de sus Juntas Auxiliares en el caso de Puebla, y de Comunidad, como se llaman a las mismas unidades políticas en el Estado de Tlaxcala. Ahora bien, ha sido el innegable e insustituible apoyo de la historia oral lo que nos permite hacer este primer esbozo de tan rica experiencia social, cultural y económica.

Considerando que los estudios históricos permiten percibir las transformaciones en el tiempo y en el espacio, el periodo central de estudio se delimita entre los años 1963-20013. Este ensayo es un primer marco de estudio para un ambicioso trabajo sobre 50 años ricos de experiencias. El reto principal está en reconstruir la geografía física y cultural de las zonas proveedoras de trabajadores, las historias de vida de estos dentro de la amplia gama de oficios involucrados en el armado de autos a lo largo de medio siglo de transformaciones, tanto de esta empresa como del país que la albergó. En cuanto a la transformación del paisaje cultural por la empresa VW en una zona que comprende los municipios de Cuautlancingo (sede de la empresa), el Municipio de Puebla, los de San Pedro Cholula, San Andrés Cholula, y Santa María Coronango en el estado de Puebla; así como los de Tenancingo, Papalotla, San Pablo del Monte, Santo Toribio Xicotzingo y Santa Inés Zacatelco el estado de Tlaxcala. Así mismo, si bien la investigación social interdisciplinaria (antropológica, sociológica, económica, estadística, demográfica, geográfica, etc.) ayuda a vislumbrar el espectro social en el corte temporal realizado, para este ensayo se ha puesto énfasis en la recolección de información tanto documental como de historia oral y estadística. Así tenemos, por ejemplo, que son las mujeres las que percibieron inmediatamente el cambio en el patrón de vida rural hacia el urbano:

Entonces ahí Cuautlancingo dio un cambio por que fueron bien pagados, todos ellos al inicio eran muy bien pagados, todos, todos los que estaban en la VOLKSWAGEN eran bien pagados, de verdad por que ya comían carne dos veces a la semana antes solo se comía una vez, por que siempre ha sido Cuautlancingo muy rico en maíz, frijol, en los cochinos, siempre tenía cada quien su cochinito, los mataban y se acaparaban de su chicharrón y lo guardaban; su rellenita, su carnita frita para que por lo menos en un mes lo freían y les dilataba. Yo me acuerdo que los echaban en unas ollitas y ahí guardaban sus chicharrones, su carne frita, la moronga como le llamaban en ese entonces; ahora le llaman rellena pero le llamaban moronga, la freían y ya comían bien. Los que no pertenecían a la gente que trabajaba en VW pues comía nopalitos, frijolitos, habitas por que estaba muy barato eso los frijolitos, el maíz, tortillas de trigo. También había tortillas de trigo en el tiempo que yo vivía, te hablo de ese tiempo que yo me crie como vestíamos; no vestíamos zapatos, fue una costumbre no tener zapatos. Escuelas nada mas para los que cursaban primaria que eran los que deveras tenían dinero, por que los padres que tenían muchos campos mejor se los llevaban al campo por que

18 Dentro de los 123 nombres de mujeres existe la duda sobre 8 ya que son nombres como "Dolores", "Guadalupe", "Cruz", "Trinidad", "Refugio" y "Ascención" que en México indistintamente se usan para hombres o mujeres. 
necesitaban gente para el campo, no para que se fueran a la escuela. Había escuelas en Puebla, aquí no, aquí solamente había primaria. ${ }^{19}$

No obstante, la dinámica de vida comunitaria interna también se verá reforzada, así como el prestigio social de las familias. No hay que pensar simplemente en una aculturación en un solo sentido:

Pos si ya participaba en las mayordomías, antes no por que estaba mas chavo, mas joven. Y cuando entró a trabajar a la VW si tuvo compromisos y muchos. Todos los que trabajaban en la VOLKSWAGEN de Cuautlancingo sabían quien era y entonces ya lo ponía en su lista, que para la fiesta de San Juan, que para la Cruz del Perdón, que para la iglesia. Entonces si, sí fue muy importante que trabajara mi esposo en la VOLKSWAGEN. ${ }^{20}$

El tránsito del trabajo poco calificado al especializado es otro proceso poco estudiado a pesar de su alta significación en los procesos de diferenciación social. Además de la cercanía geográfica y los vínculos personales necesarios para la incorporación a la gran armadora, es de notar el orden social implicado. La historia de vida de uno de los primeros trabajadores incorporados al trabajo en Volkswagen de México, es un ejemplo viviente de esos procesos.

Yo entré a trabajar de tierrero con el salario mínimo de ahí me pase a Campus Hermanos, que era una empresa constructora de estructuras, la que hizo las estructuras y este de ahí me pase a trabajar por que pues el trabajo era peligroso. En la construcción en la nave 1 que se hizo, había que trabajar hasta arriba, a no sé cuantos metros de altura que tenía la nave, para nosotros era muy alto. Las columnas que se instalaban se fijaban solo con unas llaves que les llamábamos de cola. También se movían los tragaluces por las estructuras que iban a soportar las demás trabes y bueno, andábamos ahí en las alturas. Ahí eran de 8 pulgadas mas el 4/12 pulgadas andábamos caminando en las alturas. Posteriormente entre a SIEMENS como montador; una empresa que empezó a instalar todos los tendidos de cable de alta tensión en las afueras de la nave uno y obvio estaban tendiendo líneas para la nave 2 y nave 3 , que se iniciaron casi a la vez. Ya estando en eso, conocí al que fue secretario de trabajo del sindicato de la CTM: Cristóbal León. Fuimos compañeros de trabajo en la Campus Hermanos de ahí lo conocí. Iba yo en mis ratos libres en la tarde al sindicato de la CTM a ayudarlo para recibir papeles de ingreso para la planta. Fue ahí donde nació la idea de que yo pudiera ingresar a la planta después de estar recibiendo papeles. Yo veía que entraban y yo no, y pues yo le decía ¿Por qué? Y me decía pues no, espérate a que haya un lugar. La situación de estar ganando \$45 pesos como montador con $\$ 15$ de viáticos. Cuando tuve que entrar a la planta, a un departamento que se llamaba construcción de equipos de producción, las líneas de hojalatería de acabado final, ganando $\$ 21.50$, era pos menos de la mitad de lo que yo estaba ganando pero la ilusión era de trabajar en VW y pos inicialmente entre a trabajar a VW ganando esa cantidad. ${ }^{21}$

19 Entrevista con la Sra. Alicia TEPOXTECATL ESCALANTE por Alejandra MIRANDA GARCIA. Julio 2013.

20 Ibidem

21 Entrevista realizada al S. José Toribio Miguel XICOTENCATL HERNÁNDEZ por Alejandra MIRANDA GARCIA. Agosto, 2013. 
Es de destacar igualmente el papel jugado en esta época por un sistema de valores donde el reconocimiento social se superpone al simple cálculo económico para ser partícipe de la plantilla laboral. No importaba ganar menos sino el incorporarse al grupo de trabajadores de VW que ya era distintivo regionalmente.

Los procesos de asimilación de una nueva cultura de trabajo son igualmente significativos como en este ejemplo:

\begin{abstract}
¿Alguna anécdota personal?
José Nava Tenorio: Lo único es haberse juntado con los alemanes, y eso verse en las juntas ya como parte de los de calidad, para los cambios, es muy bueno. Aparte de la experiencia, pues te da mucho el que allí las cosas se tienen que hacer bien, eso es bueno. ${ }^{22}$
\end{abstract}

\title{
Otras cuestiones sociales poco exploradas
}

Ciertos actores de la vida industrial y pública son personajes perfectamente al tanto y capaces de encabezar un movimiento de modernización en beneficio real de la sociedad mexicana. La cuestión entonces está, evidentemente, en el poder evaluar los frenos, de donde vienen y como se manifiestan; como eventualmente se transforman para hacer de la "modernidad" algo incompleto o con lagunas. ¡Es évidente que México sigue siendo hasta nuestros días totalmente dependiente de la tecnología importada por todas partes ! ¿Porqué todos los esfuerzos desplegados desde la construcción de la primera fábrica textil en 1830 no han logrado algún tipo de «soberanía tecnológica»?

Siguiendo una vez más los razonamientos de A. F. Garçon no perdemos de vista el que «... la técnica, en tanto que capacidad humana (es sobre todo) la capacidad de memorizar las prácticas, su transmisión, el potentializar sus efectos, reproducirlos, incluso desviarlos, aumentarlos ... $\gg^{23}$ todo esto más que el ser un instrumento de cambio social.

En este contexto, y en el de otros casos, la innovación e introducción de nuevas técnicas, vista desde la perspectiva de las élites modernizadoras, se percibe más como un medio de dominio que como una acción con fines de alcance social, o como una contribución al futuro de la sociedad que la alberga.

\section{Conclusión}

La acelerada urbanización que transformó el antiguo pueblo de Cuautlancingo y sus alrededores en una zona conurbada de la ciudad de Puebla, es un claro ejemplo del impulso de una industrialización desvinculada del contexto y el sistema económico del país. Se escogió al pequeño pueblo de San Lorenzo Almecatla, perteneciente al municipio de Cuautlancingo, a orillas de la autopista México-Puebla, para la construcción de la que ahora es la armadora de autos más grande del continente americano, toda vez que la nueva planta podía contar en esa localización con una conexión para el suministro económico y masivo de insumos provenientes originalmente de Alemania, así como líneas de transmisión eléctrica de alta tensión que permitirá disponer de energía a costos razonables y agua de calidad obtenida de pozos artesianos construidos ex profeso. Pero estas acciones formidables no iban acompañadas de ningún programa de desarrollo regional integral. Este caso permite igualmente constatar la necesidad prevaleciente de admitir que el patrimonio industrial, como parte integrante de nuestra sociedad, no puede desarrollarse aislado de la dinámica y los antecedentes históricos y espaciales. ¿Qué puede decir el patrimonio industrial de ayer a las so-

22 Entrevista con el Sr. José Agustín Nava Tenorio. 18 de abril de 2013. Las cursivas son nuestras

23 Garçon, Anne Françoise. L'Imaginaire et la pensée technique. Un approche historique, XVIe-XXe siècle. Classiques Garnier, Histoire des techniques 3, Paris, 2012, p 33 
ciedades contemporáneas ? ¿Cuál es el espacio de lectura que le dejamos ? ¿De que manera el nuevo patrimonio industrial puede orientar la evolución de nuestros paisajes culturales? Es a todas luces innegable que el desarrollo del patrimonio industrial contemporáneo debe responder; aquí como a través del mundo, a las demandas actuales del concepto no siempre considerado de desarrollo durable, sustentable. No puede esperarse que "se llene por sí solo lo que ha creado la decisión política", esto es: recomposición urbana, la concepción arquitectónica, la economía local, el cambio en los patrones culturales y el de las necesidades de la educación a futuro. El ciudadano, su lugar y su papel, debe estar en el centro de nuestra reflexión y del ejercicio responsable de la gestión pública. ¿Porqué entonces esta labor sine quan non del sector público parece como parte de intereses de grupo y no por y para la sociedad que la sostiene? Igualmente, es necesario considerar al patrimonio industrial como parte integrante del paisaje cultural en el que ha impactado, porque este nos expresa no sólo la impronta de individualidades formidables como vimos en este caso. El nuevo patrimonio industrial, como parte innegable del paisaje cultural, es altamente durable en sus efectos, continuará hablando a las generaciones que nos seguirán, a nuestros epígonos.

El público al cual se debe el patrimonio industrial, como en otros aspectos del complejo social, es el ciudadano. Este puede ser a la vez actor y destinatario de las acciones en favor del desarrollo industrial y económico social en general. Su perspectiva y sus necesidades difieren según los casos, las regiones y los países evidentemente; según las dimensiones de las poblaciones, ciudades, o metrópolis en las cuales habitan, así como en función de la historia industrial, de la estructura social y los niveles de soberanía política y responsabilidad social de las élites de cada país.

\section{Bibliografía}

Arteaga García, Arnulfo. Integración productiva y relaciones laborales en la industria automotriz en México. Plaza y Valdéz, Universidad Autónoma Metropolitana Iztapalapa, México, 2003. Autocar, "Volkswagen Abroad", August 10, 1951.

Berumen Arellano, Sergio Alejandro. (Coord.) Cambio tecnológico e innovación en las empresas. ESIC Editorial, Madrid, 2007.

Besusán, Graciela; García, Carlos. "Entre la estabilidad y el conflicto: relaciones laborales en VW de México, proceso de trabajo y relaciones laborales en la industria automotriz en México". en Arteaga, Arnulfo. (Coord) 1992. Proceso de trabajo y relaciones laborales en la industria automotriz en México. Fundación Friedrich Ebert, México, 1992.

Castellanos, Laura. México armado 1943-1981. Editorial Era, México, 2007.

Claval, Paul. Géographie culturelle. Une nouvelle approche des sociétés et des milieux. Ed. Armand Colin, París, 2012.

Comunicación Corporativa de Volkswagen de México. 50 años dejando huella, 1964-2014 / Comunicación Corporativa de Volkswagen de México, Puebla, México. 2013. 110 p., edición ilustrada.

Erinnerungsstätte an die Zwangsarbeit auf dem Gelände des Volkswagenwerks / Introducción y edición de Hans Mommsen. Wolfsburg, Alemania: s. e., s. f., 178 p., ilustraciones.

http://www.avwc.org/castella/historiavw.htm consultado el 28 de julio de 2014.

Loaeza, Guadalupe. Los secretos de las niñas bien. Ed. Océano, México, 2012.

Mancebo, François. Développement durable. Ed. Armand Colin, Paris, 2012.

Montiel H., Yolanda. Breve historia del Sindicato Independiente de Volkswagen de México. México: Fundación Friedrich Ebert, Representación en México, 2007. 55 p. Disponible en: http:// library.fes.de/pdf-files/bueros/mexiko/05370.pdf.

Moirón Gálvez, Sara. Crónica de Ciudad Sahagún, 1972, 188 pags.

Montiel H., Yolanda. Proceso de trabajo, acción sindical y nuevas tecnologías en Volkswagen de México. Centro de Investigaciones y Estudios superiores en Antropología Social, México, 1991.

Orozco, José Luis. La revolución corporativa. Distribuciones Fontamara, México, 2004. 
Richter, Ralf. Ivan Hirst. Britischer Offizier un Manager des Volkswagenaufbaus. Alemania: s. e., 2003. 181 p. (Colección Historische Notate; cuaderno 8).

Rieger, Bernhard. The Peopel's Car: a Global History of the Volkswagen Beetle.Harvard University Press, 2013.

Rieger, Bernhard. Technology and the Culture of Modernity in Britain and Germany, 18901945. Cambridge University Press, 2005, 319 p.

Sennett, Richard. La corrosión del carácter. Las consecuencias personales del trabajo en el nuevo capitalismo. Editorial Anagrama, Barcelona, 2000.

Schreiber, Gerhard; Calderón Sotomayor, Eduardo: Schnelle, Hermann. Una Historia sin fin. Eine Geschichte ohne ende. A Never-Ending Story. Puebla, México: Volkswagen de México, 1998.

Torres Bautista, Mariano. El origen de la industrialización de Puebla. Claves Latinoamericanas-El Colegio de Puebla, 1995, México.

Volkswagen AG, Konzernkommunikation, Historische Kommunikation (edit.). Volkswagen kronik. Textos de Markus Lupa. Wolfsburg, Alemania: s. e., 2003. 181 p. (Colección Historische Notate; cuaderno 7).

Volkswagenwerk, Place of Remembrance of the History of Forced Labor for the Volkswagen Factory. Wolfburg, 2000. 